\title{
Effects of ginsenoside Rb1 on oxidative stress injury in rat spinal cords by regulating the eNOS/Nrf2/HO-1 signaling pathway
}

\author{
XINWEI LIU ${ }^{1 *}$, XIAOCHUAN GU ${ }^{2 *}$, MIAOMIAO YU ${ }^{1}$, YING ZI $^{3}$, \\ HAILONG YU ${ }^{1}$, YU WANG ${ }^{1}$, YANCHUN XIE ${ }^{1}$ and LIANGBI XIANG ${ }^{1}$
}

\author{
${ }^{1}$ Department of Orthopedics, Rescue Center of Severe Wound and Trauma of the PLA, General Hospital of \\ Shenyang Military Area Command, Shenyang, Liaoning 110016; ${ }^{2}$ Department of Orthopedics, Changhai Hospital \\ Affiliated to The Second Military Medical University, Shanghai 200433; ${ }^{3}$ Department of \\ Emergency, Hospital 463 of the PLA, Shenyang, Liaoning 110042, P.R. China
}

Received June 2, 2017; Accepted January 5, 2018

DOI: $10.3892 /$ etm.2018.6286

\begin{abstract}
The present study aimed to investigate whether ginsenoside Rb1 (G-Rbl) attenuates spinal cord injury-associated oxidative stress in rats by regulating the endothelial nitric oxide synthase eNOS/nuclear factor erythroid 2-related factor 2 (Nrf2)/heme oxygenase (HO)-1 signaling pathway. Sprague Dawley rats were randomly divided into the sham operation group (S group), spinal cord injury group (SCI group), G-Rb1 treatment group (G-Rb1 group) and SCI+G-Rb1+Inhibitor L-name group (L-name group). The posterior limb function was evaluated via the Basso, Beattie and Bresnahan scoring method. The levels of superoxide dismutase (SOD), malondialdehyde (MDA), catalase (CAT) and glutathione (GSH) in serum were measured by ELISA. The pathological changes in the spinal cord were observed by $\mathrm{H} \& \mathrm{E}$ staining. Reverse transcription-quantitative polymerase chain reaction and western blot analyses were used to detect eNOS, phosphorylated (p)-eNOS, heat shock protein (HSP)90, Nrf2 and NAD(P)H quinone dehydrogenase 1 (Nqo1) at the mRNA and protein level. Immunohistochemistry was used to detect the expression of Nrf2 and p-eNOS. Compared with the S group, the scores of spinal cord function in the SCI group were significantly lower, and the levels of MDA were significantly increased, while the levels of SOD, CAT and GSH protein in spinal cord were significantly decreased $(\mathrm{P}<0.05)$. The spinal cord tissue exhibited hemorrhage, neuronal degeneration/necrosis, as well as mononuclear cell and lymphocyte
\end{abstract}

Correspondence to: Dr Liangbi Xiang, Department of Orthopedics, Rescue Center of Severe Wound and Trauma of the PLA, General Hospital of Shenyang Military Area Command, 83 Wenhua Road, Shenyang, Liaoning 110016, P.R. China

E-mail: xiangliangbils@126.com

*Contributed equally

Key words: spinal cord injury, ginsenoside Rb1, oxidative stress, endothelial nitric oxide synthase, nuclear factor E2-related factor 2, antioxidant response element infiltration. The eNOS, HSP90, Nrf2, Nqo1 and HO-1 mRNA levels were decreased $(\mathrm{P}<0.05)$. Compared with those in the SCI group, the spinal cord function score in the G-Rbl group were significantly higher and the serum MDA content was significantly decreased, while the activity of SOD, CAT and GSH was significantly increased $(\mathrm{P}<0.05)$. The degeneration/necrosis of spinal cord neurons was attenuated, inflammatory cell infiltration was significantly reduced and the levels of eNOS, HSP90, Nrf2, Nqo1 and HO-1 were significantly upregulated $(\mathrm{P}<0.05)$. In the group that was administered the eNOS inhibitor L-name, the levels of eNOS, HSP90, Nrf2, Nqo1 and HO-1 were significantly decreased. In conclusion, G-Rb1 attenuates oxidative stress in injured spinal cords. The mechanism may at least in part involve the eNOS/Nrf2/HO-1 pathway.

\section{Introduction}

Spinal cord injury (SCI) is the most serious complication of spinal injury. It frequently leads to dysfunction of the limbs below the injury segment, with high incidence and morbidity, but low mortality. SCI is also often associated with a younger onset and high cost as patients may be unable to return to their original health, which may affect their economic productivity and also result in a high social cost $(1,2)$. The short- and long-term effects of modern medical treatments are not ideal. SCI severely impairs the quality of life and brings a heavy economic burden to society and the families of affected patients. Identification of means to reduce SCI and promote post-operative rehabilitation is one of the hotspots in current surgical research $(3,4)$. SCI triggers secondary injury through a series of physiological and biochemical mechanisms, including oxidative stress, excessive release of excitatory amino acids and inflammatory response, so that lesions appear in intact tissue around initial lesions, which further deepens the severity and expands the scope of the injury (5). Among these factors, oxidative stress may cause an imbalance between reactive oxygen species and the anti-oxidant system, which has an important role in the secondary injury component of SCI (6). Prevention of oxidative stress to reduce the degree of secondary injury has become a potential strategy for the treatment of SCI (7). 
Ginsenoside $\mathrm{Rb} 1(\mathrm{G}-\mathrm{Rb} 1)$ is mainly derived from the stem root and flower bud of Panax quinquefolius and Panax notoginseng. A previous study indicated that G-Rb1 has anti-oxidant effects, scavenges free radicals and improves the body's immunity as mechanisms due to which it has been used for the treatment of various traumatic diseases $(8,9)$. Cheng et al (10) demonstrated that $\mathrm{G}-\mathrm{Rb} 1$ reduces prostaglandin $\mathrm{E} 2, \mathrm{NO}_{2}$, matrix metalloproteinase-13, cyclooxygenase-2, inducible nitric oxide synthase (NOS), caspase-3 and poly(ADP ribose) polymerase levels, thus preventing the interleukin-1-induced inflammatory response and apoptosis of human articular chondrocytes. In study using a hydrogen peroxide-induced human umbilical vein endothelial cell model of aging, Liu et al (11) revealed that $\mathrm{G}-\mathrm{Rb} 1$ promotes the production of intracellular superoxide dismutase (SOD), reduces the content of the lipid peroxidation product malondialdehyde (MDA) and protects cells against oxidative stress-induced senescence. G-Rb1 adjusts the immune balance and scavenges free radicals, but it has remained elusive whether it attenuates the oxidative stress injury of the spinal cord through its anti-oxidant effect.

Nuclear factor erythroid 2-related factor 2 (Nrf2)/heme oxygenase (HO)-1 is considered to be the most important anti-oxidant pathway. The key role of Nrf2/HO-1 in controlling foreign bodies and oxidative damage has been confirmed in the digestive, circulatory and nervous system, as well as in diseases affecting the immune system. Activation of this pathway triggers the production of corresponding anti-oxidant enzymes and phase-II drug metabolism enzymes, thereby enhancing the ability of cells to remove reactive oxygen species to maintain a redox balance and reduce oxidative damage. It has been reported that G-Rb1 improves organ injury induced by intestinal ischemia-reperfusion in C57BL/6J mice by activating the Nrf2/HO-1 pathway (12). However, it has remained elusive whether G-Rb1 exerts its protective effect against secondary SCI via the endothelial (e)NOS/Nrf2/HO-1 signaling pathway. The aim of the present study was to explore the specific implication of the eNOS/Nrf2/HO-1 pathway in the effect of G-Rb1 on oxidative stress injury of rat spinal cords as a possible mechanism of its protective action.

\section{Materials and methods}

Animals. Sprague Dawley rats ( $\mathrm{n}=40 ; 7$ weeks old; $50 \%$ male and 50\% female; weight, $220-260 \mathrm{~g}$ ), were provided by the Experimental Animal Division of the General Hospital of Shenyang Military Area Command [Shenyang, China, rodent application license no. SYXK (Jun) 20120003; rodent production license no. SCXK (Army)20120001]. The experiment was approved by the Experimental Animal Ethics Committee of the General Hospital of Shenyang Military Area Command (Shenyang, China). Animals were housed at a constant temperature $\left(22 \pm 1^{\circ} \mathrm{C}\right)$ with $50 \%$ humidity in a $12 \mathrm{~h}$ light/dark cycle. The rats had ad libitum access to food and autoclaved water. Animals of different sex were kept in separate cages.

Establishment of rat SCI model. The rat SCI model was established using Allen's modified method (force, $25 \mathrm{~g} / \mathrm{cm}$; impact force, $10 \mathrm{~g}$; fall height, $5 \mathrm{~cm})(13,14)$. All animals were fasted for $12 \mathrm{~h}$ and deprived of water for $4 \mathrm{~h}$ prior to surgery. Animals were maintained warm during surgery. Rats were anesthetized by intraperitoneal injection of $2 \%$ pentobarbital sodium $(45 \mathrm{mg} / \mathrm{kg}$ ) and then fixed in the prone position on the operating table. A $2-\mathrm{cm}$ incision was made along the posterior midline of the spine and the muscle was bluntly isolated, followed by laminectomy. The T10 chest segment was exposed and injured with a heavy hammer (designed using Allen's modified method; 25 gram $\mathrm{cm}$ force; impact force, $10 \mathrm{~g}$; fall height, $5 \mathrm{~cm}$ ) with a bottom diameter of $1.5 \mathrm{~mm}$, resulting in moderate SCI $(15,16)$. The heavy hammer was removed immediately after impact and the wound was sutured layer by layer.

Groups and treatments. Rats were randomly divided into the sham operation group (S group), SCI group, G-Rb1 treatment group (G-Rb1 group) and SCI+G-Rb1+inhibitor L-name group (L-name group), with 10 rats in each group (50\% male and $50 \%$ female in each group). Rats in the $\mathrm{S}$ group received laminectomy only; rats in G-Rbl group were intraperitoneally injected with G-Rb1 (10 mg/kg; Sigma-Aldrich; Merck KGaA, Darmstadt, Germany) at 30 min after modeling and then daily for 7 days. Rats in the SCI group were given an intraperitoneal injection of an equal amount of normal saline and rats in the L-name group were intraperitoneally injected with G-Rb1 $(10 \mathrm{mg} / \mathrm{kg})$ and given a tail vein injection of eNOS inhibitor L-name at $30 \mathrm{~min}$ post modeling and then daily for 7 days (7 mg/kg; cat. no. 51298-62-5; MedChemExpress, Monmouth Junction, NJ, USA).

Basso, Beattie and Bresnahan (BBB) locomotor rating scale. The motor function of the hind limbs was evaluated using the BBB method as previously described (17). The animals were observed according to the standard BBB grading standards and the recovery of motor function in the hind limbs was recorded. All observations were performed simultaneously. Scoring criteria were as follows: 0-7 points, joint activity; 8-13 points, gait and coordination function; $14-21$ points, claw movement. The maximum score was 21 points and hind limb paralysis was scored as 0 points.

Sample collection and testing. The rats were euthanized using an overdose of $2 \%$ pentobarbital sodium $(120 \mathrm{mg} / \mathrm{kg}$; intraperitoneal injection) at $24 \mathrm{~h}$ after the last injection. Blood samples taken from the abdominal aorta were centrifuged and stored at $-80^{\circ} \mathrm{C}$. Spinal cord tissue was harvested from the injured area, of which one part was fixed in $10 \%$ formalin, and another part was stored in liquid nitrogen.

ELISA. The changes of SOD (cat. no. SES134Ra), MDA (cat. no. CEA597Ge), glutathione (GSH; cat. no. CEA294Ge) (all Wuhan USCN Business Co., Ltd., Wuhan, China) and catalase (CAT; cat. no. CSB-E13439r; Cloud-Clone Corp., Katy, TX, USA) in serum were detected with an ELISA kit according to the manufacturer's protocols. The optical density at $450 \mathrm{~nm}$ was measured using a microplate reader (Bio-Rad 680; Bio-Rad Laboratories, Inc., Hercules, CA, USA) and the concentration was determined using a standard curve.

$H \& E$ staining. Tissues were fixed in $10 \%$ formaldehyde for $24 \mathrm{~h}$ at room temperature and then decalcified, dehydrated and permeabilized using 50\% xylene for $1 \mathrm{~h}$ and $100 \%$ xylene for $2 \mathrm{~h}$. The tissues were embedded in wax and sliced into 
Table I. Primer sequences used for polymerase chain reaction.

\begin{tabular}{lll}
\hline Gene & Upstream primer sequence $\left(5^{\prime}-3^{\prime}\right)$ & Downstream primer sequence $\left(5^{\prime}-3^{\prime}\right)$ \\
\hline eNOS & ACCGCCACACAGTAAATCCA & TGCCAACAGGAAGCTGAGAG \\
HSP90 & ACCAAGTCCCAGCTCACAAA & TGGGGAGAAAGCAACCACTG \\
$\mathrm{Nrf} 2$ & ATGAGTCGCTTGCCCTGG & CTTGTTTCCGTATTAAG \\
$\mathrm{Nqo} 1$ & CTTGCTTTCCATCACCACCG & GACGCTTCTTCCACCCTTCC \\
$\mathrm{HO}-1$ & AGCATGTTCCCAGGATG & GCTCAATGTTGAGCACA \\
$\beta$-actin & CTG TCCCTGTATGCCTCT & ATGTCACGCACGATTTCC \\
\hline
\end{tabular}

eNOS, endothelial nitric oxide synthase; Nrf2, nuclear factor erythroid 2-related factor 2; HSP, heat shock protein; Nqo1, NAD(P)H quinone dehydrogenase $1 ; \mathrm{HO}$, heme oxygenase.

5- $\mu$ m-thick sections using a microtome. All of the following steps were performed at room temperature. Sections were then dewaxed using xylene I for $15 \mathrm{~min}$ and xylene II for $15 \mathrm{~min}$, hydrated with absolute ethanol for $5 \mathrm{~min}, 90 \%$ ethanol for $2 \mathrm{~min}$ and $70 \%$ ethanol for $2 \mathrm{~min}$. They were subsequently mounted with $10 \%$ hematoxylin for $10 \mathrm{~min}$, differentiated with $1 \%$ hydrochloric acid and ethanol for 3-5 sec and stained with $0.5 \%$ eosin for $1 \mathrm{~min}$. Then they were dehydrated in an alcohol gradient with xylene, cleared and mounted. Using a light microscope, pathological changes in the spinal cord following ginsenoside Rb1 treatment were observed.

Reverse transcription-quantitative polymerase chain reaction (RT-qPCR) analysis. Primers were designed according to the sequences of the eNOS, heat shock protein (HSP)90, Nrf2, $\mathrm{HO}-1$ and $\mathrm{NAD}(\mathrm{P}) \mathrm{H}$ quinone dehydrogenase 1 (Nqo1) genes listed in GenBank. The primers were then synthesized in Shanghai Sangon Biotechnology Co., Ltd. (Shanghai, China) and the sequences are listed in Table I.

Spinal cord RNA was extracted using TRIzol (cat. no. 15596018; Invitrogen ${ }^{\mathrm{TM}}$; Thermo Fisher Scientific, Inc., USA) and reverse transcribed into complementary DNA (cat. no. 4387406, Invitrogen ${ }^{\mathrm{TM}}$, Thermo Fisher Scientific, Inc.) according to the manufacturer's protocol. The composition of the reverse transcription mixture was as follows: $10 \mu 12 \mathrm{X}$ RT buffer mix, $1 \mu 1$ 20X RT enzyme mix, $2 \mu 1$ RNA sample and $7 \mu \mathrm{l}$ nuclease-free $\mathrm{H}_{2} \mathrm{O}$. The reaction was performed at $37^{\circ} \mathrm{C}$ for $60 \mathrm{~min}$. The reaction was stopped by heating to $95^{\circ} \mathrm{C}$ for $5 \mathrm{~min}$ and holding at $4^{\circ} \mathrm{C}$. This was followed by detection with a real-time PCR kit (iQ5; Bio-Rad Laboratories, Inc.) in a real-time PCR system (RR820A; Takara Bio, Inc., Otsu, Japan). The following thermocycling conditions were used for RT-qPCR: Initial denaturation at $95^{\circ} \mathrm{C}$ for $30 \mathrm{sec} ; 40$ cycles of PCR at $95^{\circ} \mathrm{C}$ for $5 \mathrm{sec}$ and $60^{\circ} \mathrm{C}$ for $30 \mathrm{sec}$; with a final dissociation stage at $95^{\circ} \mathrm{C}$ for $15 \mathrm{sec}, 60^{\circ} \mathrm{C}$ for $1 \mathrm{~min}$ and $95^{\circ} \mathrm{C}$ for $15 \mathrm{sec}$. The relative gene expression data was analyzed with the $2^{\Delta \Delta \mathrm{Cq}}$ method (18).

Western blot analysis. Total tissue protein was extracted with a protein extraction kit (cat. no. 78510; Thermo Fisher Scientific, Inc.) and the protein concentration was determined by a bicinchoninic acid protein quantification kit (cat. no. 23229; Thermo Scientific, Inc.). Protein samples (30 $\mu \mathrm{g} /$ lane) were subjected to $10 \%$ SDS-PAGE and transferred

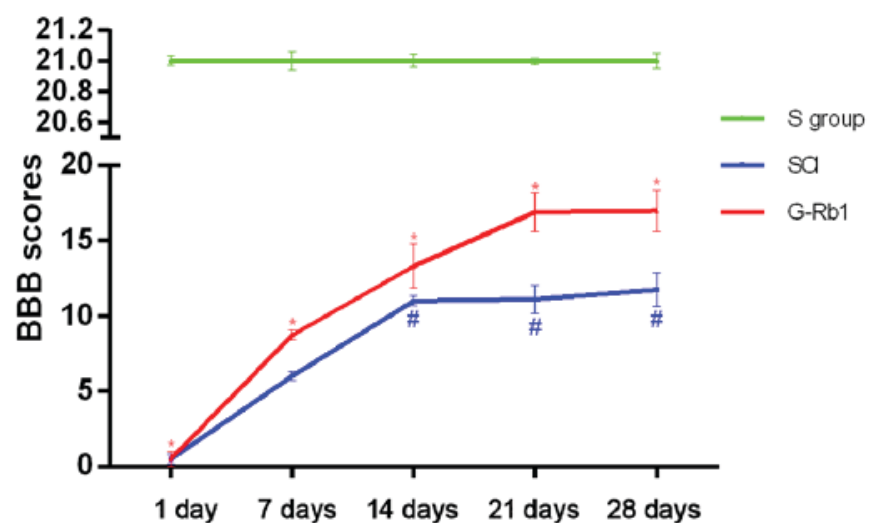

Figure 1. BBB scores after at different days after SCI. * $\mathrm{P}<0.05$ vs. S group; ${ }^{\#} \mathrm{P}<0.05$ vs. SCI group. SCI, spinal cord injury; BBB, Basso, Beattie and Bresnahan; G-Rb1, ginsenoside Rb-1; S, sham.

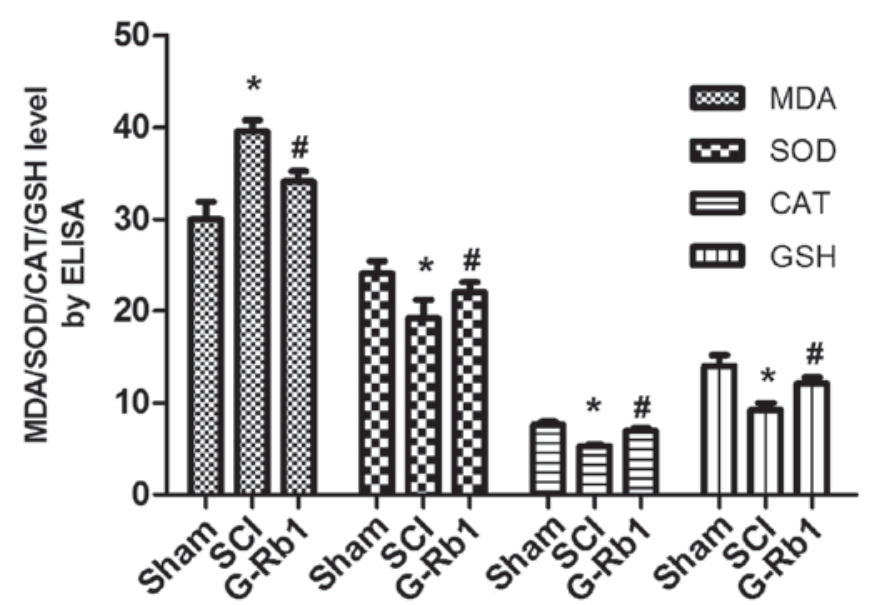

Figure 2. SCI-induced changes in the serum levels of MDA, SOD, CAT and GSH as determined by ELISA. ${ }^{*} \mathrm{P}<0.05$ vs. $\mathrm{S}$ group; ${ }^{\#} \mathrm{P}<0.05$ vs. SCI group. S, sham; SCI, spinal cord injury; MDA, malondialdehyde; SOD, superoxide dismutase; CAT, catalase; GSH, glutathione; G-Rb1, ginsenoside Rb-1.

to polyvinylidene difluoride membranes (cat. no. IB24002, Invitrogen; Thermo Fisher Scientific, Inc.). The samples were incubated with $5 \%$ non-fat powdered milk with $100 \mathrm{ml}$ Tris-buffered saline with Tween-20 for $1 \mathrm{~h}$, followed by incubation with primary antibodies to eNOS (mouse monoclonal 

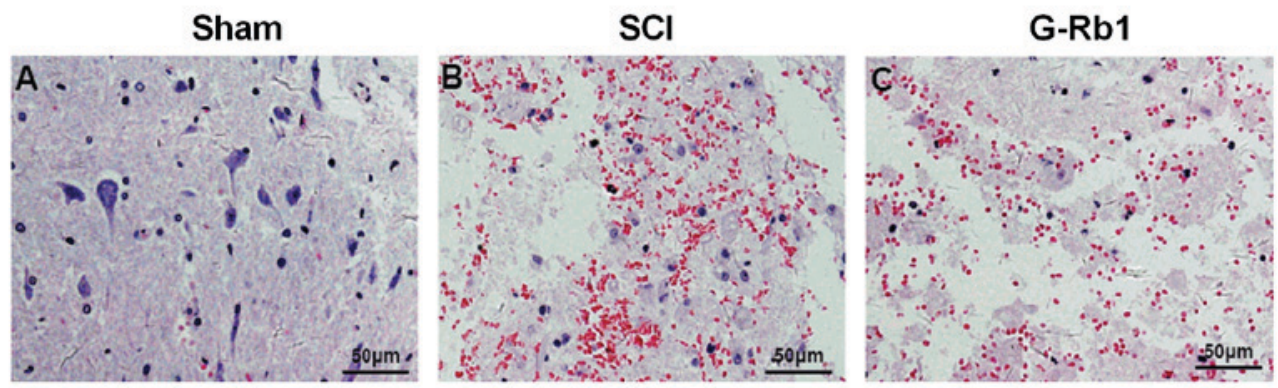

Figure 3. Morphological changes in the spinal cord after G-Rb1 treatment (A) Sham group (B) SCI group, the tissue displayed increased haemorrhage, neuronal degeneration/necrosis and mononuclear cell/lymphocyte infiltration compared with the Sham group. (C) G-Rb1 group, the hemorrhage, neuronal degeneration/necrosis, and mononuclear cell/lymphocyte infiltration was attenuated compared with the SCI group (scale bar, 50 $\mu \mathrm{m}$ ). G-Rb1, ginsenoside Rb-1; SCI, spinal cord injury.

antibody; 1:1,500 dilution; cat. no. ab76198), HSP90 (1:10,000 dilution; cat. no. ab203126), Nrf2 (1:1,000 dilution; cat. no. ab137550), Nqo1 (1:2,000 dilution; cat. no. ab217302) and HO-1 (1:1,000 dilution; cat. no. ab82585) at $4^{\circ} \mathrm{C}$ overnight. Subsequently, membranes were incubated with goat anti-mouse immunoglobulin (Ig) G H\&L horseradish peroxidase (HRP) conjugated (1:2,000 dilution; cat. no. ab6789) or goat anti-rabbit IgG H\&L HRP conjugated (1:2,000 dilution; cat. no. ab205718) secondary antibodies at $4^{\circ} \mathrm{C}$ for $2 \mathrm{~h}$. All primary and secondary antibodies were purchased from Abcam (Cambridge, MA, USA) Subsequently, samples were developed with the enhanced chemiluminescence method using Novex ${ }^{\mathrm{TM}}$ ECL Chemiluminescent Substrate Reagent kit (cat. no. WP20005; Invitrogen; Thermo Fisher Scientific, Inc.) for 1-2 min and quantified with an automatic chemiluminescence imaging system (Tanon 5200; Shanghai Tianneng Technology Co., Ltd., Shanghai, China).

Immunohistochemical staining. Spinal cord tissues were dewaxed with xylene, dehydrated with a gradient series of ethanols, incubated with $3 \%$ hydrogen peroxide for $20 \mathrm{~min}$ at room temperature and washed three times with PBS for $5 \mathrm{~min}$ each. The tissue was then blocked with $10 \%$ goat serum (cat. no. ab7481; Abcam) for $30 \mathrm{~min}$ at room temperature. The cells were incubated with antibodies against p-eNOS (1:400 dilution; cat. no. bs-13074R; BIOSS, Beijing, China) Nrf2 (1:100 dilution) in a humidified chamber at $4^{\circ} \mathrm{C}$ overnight, followed by goat anti-rabbit IgG H\&L HRP conjugated (1:2,000 dilution) for $30 \mathrm{~min}$ at room temperature. Immunoreactivity was then visualized with diaminobenzidine (DAB; cat. no. DA1015) and haematoxylin (cat. no. G1140) (both Beijing Solarbio Science \& Technology Co., Ltd., Beijing, China) counterstaining was applied. The samples were observed under a light microscope (magnification, x200).

Statistical analysis. Values are expressed as the mean \pm standard deviation. Student's t-test was used to assess differences between 2 groups. One-way analysis of variance followed by Bonferroni's post-hoc test was used assess differences among $>2$ groups. All pairwise P-values are two-sided. $\mathrm{P}<0.05$ was considered to indicate a statistically significant difference. All data were statistically analyzed using SPSS version 19.0 software (IBM Corp., Armonk, NY, USA).

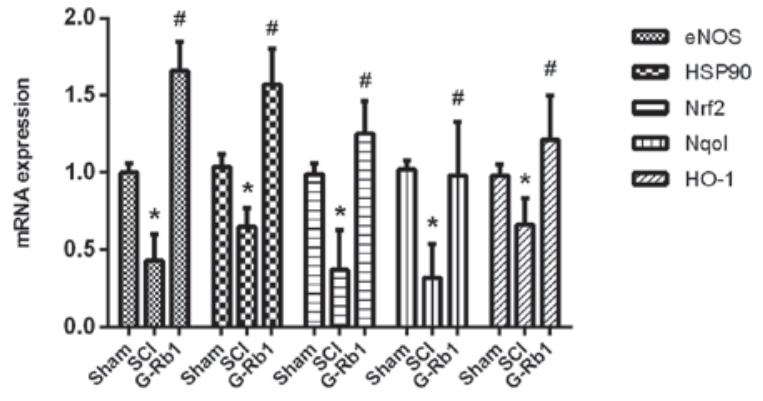

Figure 4. mRNA expression of eNOS, HSP90, Nrf2, Nqo1 and HO-1 after ginsenoside Rb1 treatment determined by reverse transcription-quantitative polymerase chain reaction. ${ }^{*} \mathrm{P}<0.05$ vs. $\mathrm{S}$ group; ${ }^{\text {}} \mathrm{P}<0.05$ vs. SCI group. $\mathrm{S}$, sham; SCI, spinal cord injury; HSP, heat shock protein; HO, heme oxygenase; eNOS, endothelial nitric oxide synthase; Nrf2, nuclear factor erythroid 2-related factor 2; G-Rb1, ginsenoside Rb-1; Nqo1, NAD(P)H quinone dehydrogenase 1 .

\section{Results}

G-Rbl improves the hind limb motor function of SCI rats. All rats were released from the cage and conditioned in an open space for $5 \mathrm{~min}$, and the BBB test was performed and recorded using camera monitoring. The evaluators were blinded to the experimental grouping and treatment. The total score ranged from 0-21 points. As presented in Fig. 1, on day 1, none of the rats in the SCI and G-Rb1 groups scored higher than 2 points, indicating that the spinal cord was seriously damaged and hind limb motor dysfunction was obvious. On day 7 the motor function of the hind limbs in the G-Rb1 group was significantly improved, with higher scores than those in the $\mathrm{SCI}$ group $(\mathrm{P}<0.05)$.

$G$-Rbl attenuates SCI-induced changes in the serum content of $S O D, M D A, C A T$ and $G S H$. Compared with those in the $\mathrm{S}$ group, the levels of MDA were significantly increased $(\mathrm{P}<0.05)$, and the levels of SOD, CAT and GSH were significantly decreased in the SCI group $(\mathrm{P}<0.05)$. G-Rb1 significantly decreased the levels of MDA, and increased the levels of SOD, CAT and GSH compared with those in the SCI group ( $\mathrm{P}<0.05$; Fig. 2).

G-Rbl attenuates SCI-induced histopathological changes. Compared with the $\mathrm{S}$ group, the spinal cord tissue displayed 

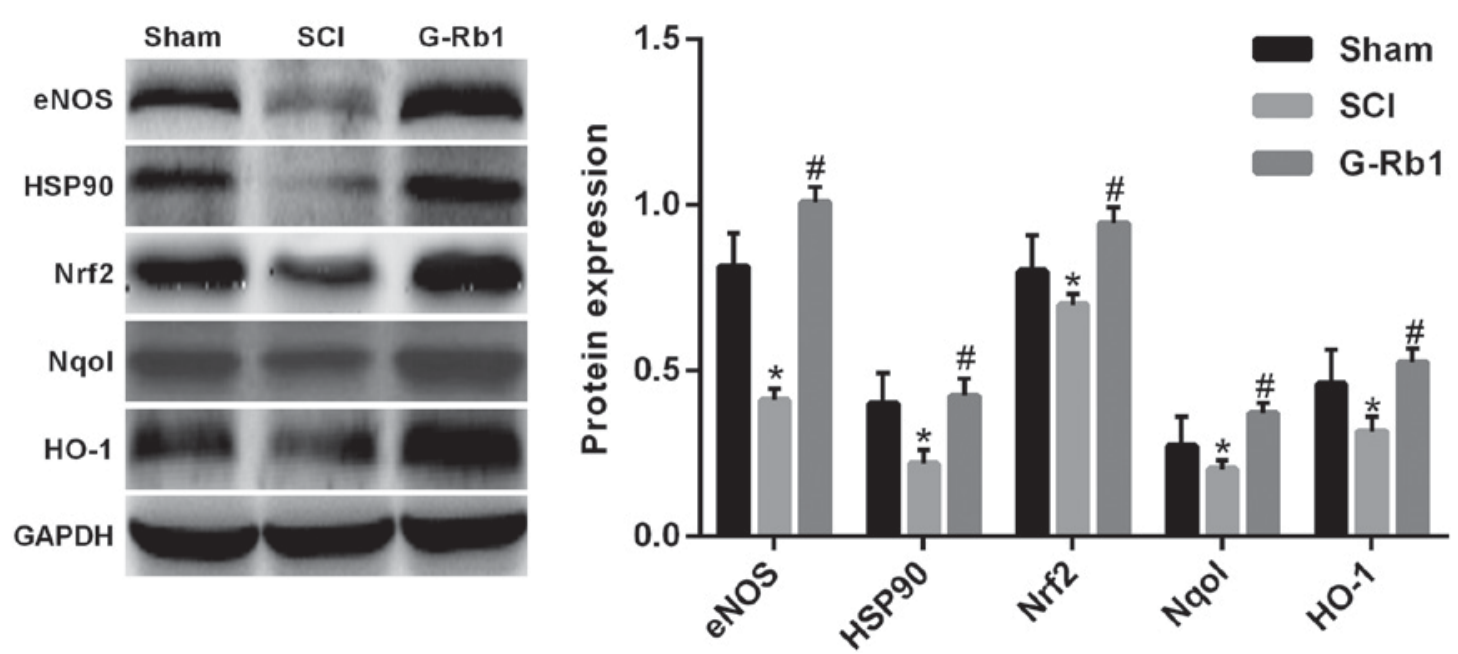

Figure 5. Expression of eNOS, HSP90, Nrf2, Nqo1 and HO-1 protein determined by western blot analysis. ${ }^{*} \mathrm{P}<0.05$ vs. S group; ${ }^{~ P}<0.05$ vs. SCI group. S, sham; SCI, spinal cord injury; HSP, heat shock protein; HO, heme oxygenase; eNOS, endothelial nitric oxide synthase; Nrf2, nuclear factor erythroid 2-related factor 2; G-Rb1, ginsenoside Rb-1; Nqo1, NAD(P)H quinone dehydrogenase 1.
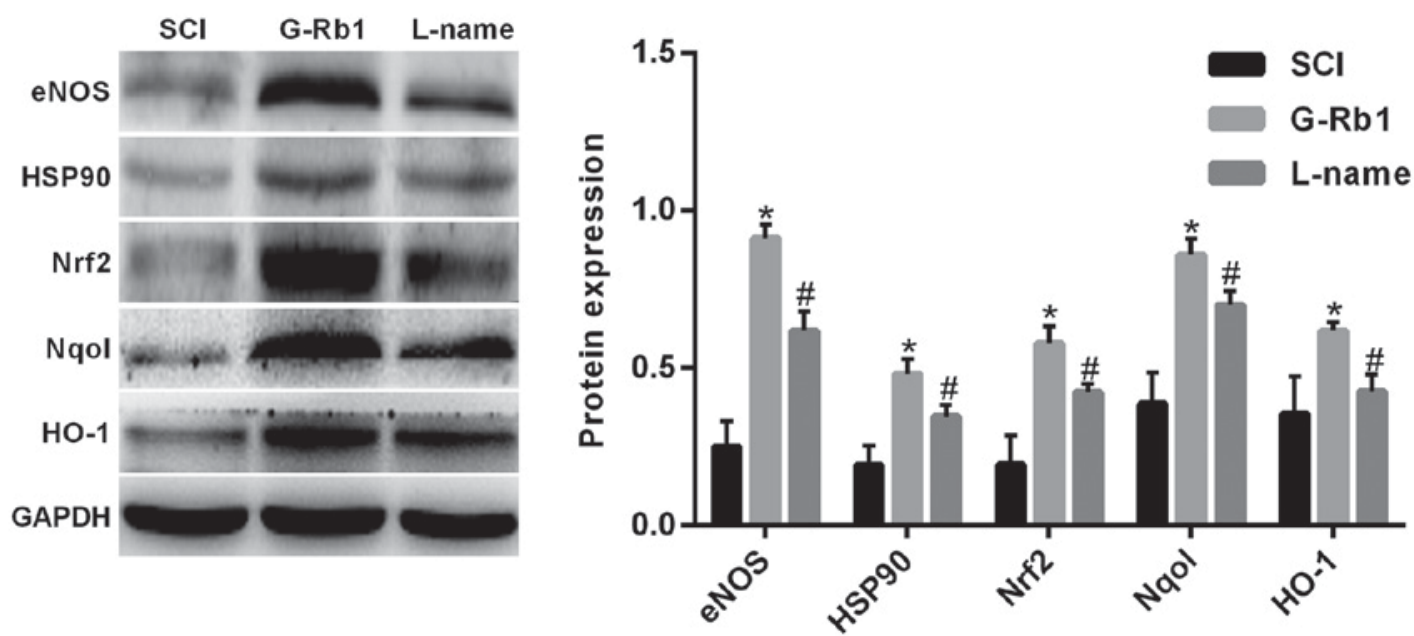

Figure 6. Effect of L-name on the expression of eNOS, HSP90, Nrf2, Nqo1 and HO-1 in a rat model of SCI. "P<0.05 vs. S group; ${ }^{\text {P }}<0.05$ vs. SCI group. S, sham; SCI, spinal cord injury; HSP, heat shock protein; HO, heme oxygenase; eNOS, endothelial nitric oxide synthase; Nrf2, nuclear factor erythroid 2-related factor 2; G-Rb1, ginsenoside Rb-1; Nqo1, NAD(P)H quinone dehydrogenase 1.

hemorrhage, neuronal degeneration/necrosis, as well as mononuclear cell and lymphocyte infiltration in the SCI group. Of note, G-Rb1 attenuated the hemorrhage, neuronal degeneration/necrosis, as well as mononuclear cell and lymphocyte infiltration compared with that in the SCI group (Fig. 3).

G-Rb1 modulates SCI-induced expression of NOS, HSP90, Nrf2, Nqol and HO-1 mRNA. Compared with that in the S group, the expression of NOS, HSP90, Nrf2, Nqo1 and HO-1 mRNA in spinal cord tissue of the SCI group was significantly decreased $(\mathrm{P}<0.05)$. However, G-Rb1 significantly increased NOS, HSP90, Nrf2, Nqo1 and HO-1 mRNA expression levels compared with that in the SCI group ( $\mathrm{P}<0.05$; Fig. 4).

G-Rbl modulates SCI-induced expression of NOS, HSP90, $\mathrm{Nrf2,} \mathrm{Nqol}$ and HO-1 protein. Compared with that in the $\mathrm{S}$ group, the expression of eNOS, HSP90, Nrf2, Nqo1 and HO-1 protein in the spinal cord tissue of the SCI group was significantly decreased $(\mathrm{P}<0.05)$; however, G-Rb1 significantly increased eNOS, HSP90, Nrf2, Nqo1 and HO-1 protein expression in the spinal cord compared with that in the SCI group $(\mathrm{P}<0.05)$ (Fig. 5). In order to further demonstrate that the protective effect of G-Rb1 is dependent on the eNOS/Nrf2/anti-oxidant response element (ARE) pathway, rats subjected to SCI and receiving G-Rb1 were injected with the eNOS inhibitor L-name. The results indicated that in this L-name group, the expression of HSP90, Nrf2, Nqo1 and HO-1 was significantly decreased compared with that in the G-Rb1 group $(\mathrm{P}<0.05$; Fig. 6$)$. These results may suggest that G-Rb1 attenuates oxidative stress in the spinal cord via the eNOS/Nrf2/ARE signaling pathway.

Effect of G-Rbl on the expression of p-eNOS and Nrf2 protein post SCI. Immunohistochemical analysis indicated that compared with that in the S group, the level of p-eNOS in the spinal cord tissue of the SCI group was notably increased and 

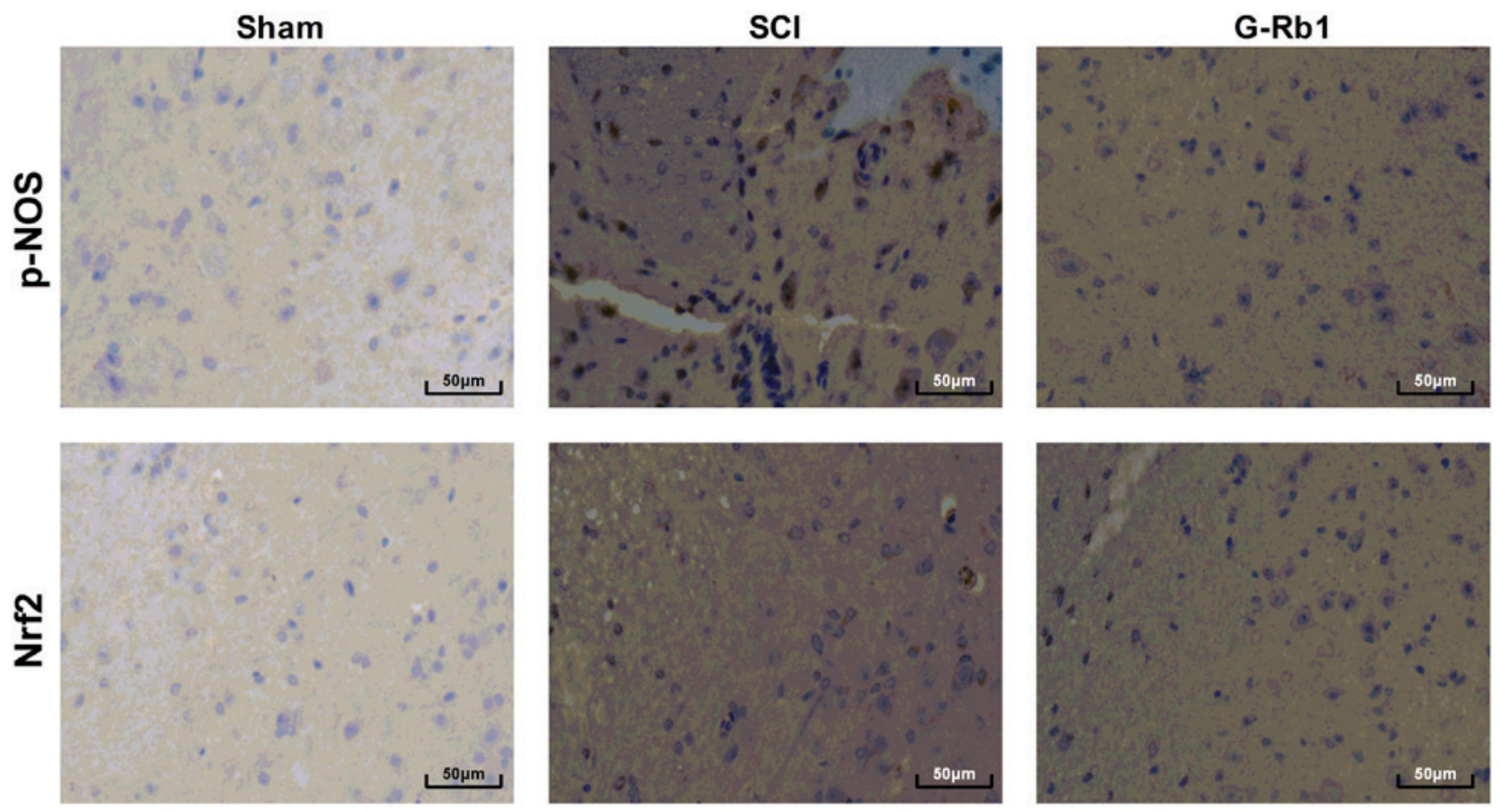

Figure 7. Expression of p-eNOS and Nrf2 protein as determined by immunohistochemistry. DAB staining and haematoxylin counterstaining were applied and immunostaining was indicated with a blue color. Scale bar, $50 \mu \mathrm{m}$. p-eNOS, phosphorylated endothelial nitric oxide synthase; Nrf2, nuclear factor erythroid 2-related factor 2; G-Rb1, ginsenoside Rb-1; SCI, spinal cord injury.

the level of Nrf2 was notably decreased. Compared with those in the SCI group the p-eNOS level was markedly increased and the Nrf2 level was markedly decreased in the G-Rb1 group (Fig. 7).

\section{Discussion}

SCI may trigger oxidative stress, change membrane permeability, and induce lysosomal disintegration and cell necrosis, resulting in secondary damage to the spinal cord. The present study indicated that compared with the control group, spinal cord function scores of SCI rats were significantly decreased, neuronal degeneration and necrosis were present, the levels of MDA in serum were significantly increased, SOD, CAT and GSH protein expression was significantly decreased, and eNOS/Nrf2 protein expression was significantly decreased. Of note, G-Rb1 significantly increased the hind limb function score, reduced the MDA content, increased SOD, CAT and GSH content, and upregulated eNOS/Nrf2 protein expression in SCI rats.

MDA is a metabolite of lipid peroxidation, and its content reflects the degree of lipid peroxidation in vivo, as well as indirectly reflects the degree of cell damage caused by oxygen free radicals. SOD and CAT are important anti-oxidant enzymes, which scavenge oxygen free radicals generated in the process of tissue and cell metabolism, thus protecting the body from oxidative stress injury, and their activity indirectly reflects the anti-oxidant capacity of the body. Therefore, it is important to monitor the activity of MDA, SOD and CAT in spinal cord tissue of SCI rats in order to observe their oxidative stress status (19). In the present study, the MDA content was significantly increased, while SOD, CAT and GSH protein expression was significantly decreased, and the levels of eNOS, p-eNOS,
HSP90, Nrf2 and Nqo1 were decreased. In the resting state, the redox sites of eNOS are occupied by caveolin (CAV)-1 and eNOS is inactivated; when the cells are stimulated by external signals, the intracellular $\mathrm{Ca}^{2+}$ concentration increases, and calmodulin and HSP90 occupy the CAV-1 binding sites on eNOS, leading to the dissociation of eNOS from CAV-1 and eNOS is activated (20-22). Nrf2 is an important transcription factor, which reduces reactive oxygen species and promotes the body's resistance to harmful external stimuli. Under physiological conditions, Kelch-like ECH-associated protein 1 (Keap1) in the cytoplasm is linked to Nrf2 to inactivate it (23-25). Upregulation of eNOS results in increased nitrosylation of Keap1. Nrf2 detaches from Keap1 and is thereby activated, and translocates into the nucleus, where it recognizes and binds to a series of AREs. As downstream events, ARE then activates the expression of certain corresponding phase-II detoxification enzyme genes, induces the expression of SOD, CAT, GSH, leading to the clearance and metabolism of free radicals (11). Nqo1 is one of Nrf2-driven downstream target genes that is involved in anti-oxidant stress injury $(26,27)$. Activated eNOS regulates the Nrf2/HO-1 signaling pathway, increases the expression of anti-oxidant enzymes, and reduces the incidence of cardiovascular disease. Studies have indicated that small doses of tert-butyl hydroquinone activate $\mathrm{Nrf} 2$, upregulate the expression of anti-oxidant enzymes and regulate oxidative damage of nerve cells $(28,29)$. In the early stage of fluorosis, expression of anti-oxidant enzymes, including SOD, CAT and GSH-Px is upregulated by activation of the Nrf2/HO-1 pathway, enhancing the ability of cells to resist oxidative stress and reduce the production of oxygen free radicals (30). Sulforaphane and carnosic acid contribute to the activation of $\mathrm{Nrf} 2 / \mathrm{HO}-1$, effectively reducing the binding of 
4-hydroxynonenal to mitochondria, and inhibiting oxidative stress (25). Therefore, the Nrf2-mediated anti-oxidant response has a crucial role in functional recovery after SCI $(31,32)$.

The pharmacological effects of ginseng include regulation of the central nervous system, enhancement of physical strength, reduction of fatigue and improvement of the metabolism. G-Rb1 as the major active component of ginseng scavenges oxygen free radicals, blocks calcium overload in nerve cells, improves energy metabolism and maintains neuronal cell integrity $(33,34)$. Sun et al $(33)$ have reported that after intestinal ischemia-reperfusion, the renal MDA content in rats was increased and SOD levels were decreased, while G-Rb1 can reduced oxidative stress injury by activating the Nrf2/HO-1 pathway. Hwang et al (34) have demonstrated that G-Rb1 effectively reduces 6-hydroxydopamine-induced oxidative stress injury in human dopaminergic cells by activating Nrf2/HO-1. In the present study, G-Rb1 significantly increased the hind limb function score, decreased the content of MDA, increased the content of SOD, CAT and GSH, and enhanced the expression of eNOS, HSP90, Nrf2 and Nqo1 protein in SCI rats. The mechanisms may include scavenging of free radicals, improvement of anti-oxidant enzyme activity and blocking of lipid peroxidation to protect cells from oxidative stress injury, thus maintaining the physiological function of the spinal cord tissue.

In conclusion, the present study demonstrated that G-Rb1 significantly increased the hind limb function score, decreased the MDA content, increased the SOD, CAT and GSH content, and upregulated eNOS/Nrf2 protein expression in SCI rats, which exerted an obvious protective effect against oxidative stress injury, and the underlying mechanism may be associated with the eNOS/Nrf2/HO-1 pathway.

\section{Acknowledgements}

Not applicable.

\section{Funding}

This study was supported by the Scientific and technological project in Liaoning Province (No. 2014225012).

\section{Availability of data and materials}

The datasets used and/or analyzed during the current study are available from the corresponding author on reasonable request.

\section{Authors' contributions}

XL, XG and LX conceived and designed the study, acquired data, interpreted the results and drafted the manuscript. LX also contributed to the acquisition of funding and support. XL, MY, YZ, HY and YW performed the experiments. LX and YX analyzed the data. All authors read and approved the final manuscript.

\section{Ethics approval and consent to participate}

The study was approved by the Experimental Animal Ethics Committee of the General Hospital of Shenyang Military Area Command (Shenyang, China).

\section{Patient consent for publication}

Not applicable.

\section{Competing interests}

The authors declare that they have no competing interests.

\section{References}

1. Mitchell R, Harvey L, Stanford R and Close J: Health outcomes and costs of acute traumatic spinal injury in New South Wales, Australia. Spine J 2017 (Epub Ahead of Print).

2. Moshi H, Sundelin G, Sahlen KG and Sorlin A: Traumatic spinal cord injury in the north-east Tanzania-describing incidence, etiology and clinical outcomes retrospectively. Global health Action 10: 1355604, 2017.

3. Bothig R, Fiebag K, Thietje R, Faschingbauer M and Hirschfeld S: Morbidity of urinary tract infection after urodynamic examination of hospitalized SCI patients: The impact of bladder management. Spinal Cord 51: 70-73, 2013.

4. Pannek J: Editorial Note on: Morbidity of urinary tract infection after urodynamic examination of hospitalized SCI patients: The impact of bladder management. Spinal Cord: Sep 18, 2012 (Epub Ahead of Print).

5. Borges TJ, Lang BJ, Lopes RL and Bonorino C: Modulation of alloimmunity by heat shock proteins. Front Immunol 7: 303, 2016.

6. Ren J, Fan C, Chen N, Huang J and Yang Q: Resveratrol pretreatment attenuates cerebral ischemic injury by upregulating expression of transcription factor $\mathrm{Nrf} 2$ and $\mathrm{HO}-1$ in rats. Neurochem Res 36: 2352-2362, 2011.

7. Chini MG, Malafronte N, Vaccaro MC, Gualtieri MJ, Vassallo A, Vasaturo M, Castellano S, Milite C, Leone A, Bifulco G, et al: Identification of limonol derivatives as heat shock protein 90 (Hsp90) Inhibitors through a multidisciplinary approach. Chemistry 22: 13236-13250, 2016.

8. Dong X, Zheng L, Lu S and Yang Y: Neuroprotective effects of pretreatment of ginsenoside Rb1 on severe cerebral ischemia-induced injuries in aged mice: Involvement of anti-oxidant signaling. Geriatr Gerontol Int 17: 338-345, 2017.

9. Chen W, Guo Y, Yang W, Zheng P, Zeng J and Tong W: Involvement of connexin40 in the protective effects of ginsenoside Rb1 against traumatic brain injury. Cell Mol Neurobiol 36: 1057-1065, 2016.

10. Cheng W, Wu D, Zuo Q, Wang Z and Fan W: Ginsenoside Rb1 prevents interleukin-1 beta induced inflammation and apoptosis in human articular chondrocytes. Int Orthop 37: 2065-2070, 2013.

11. Liu DH, Chen YM, Liu Y, Hao BS, Zhou B, Wu L, Wang M, Chen L, Wu WK and Qian XX: Rb1 protects endothelial cells from hydrogen peroxide-induced cell senescence by modulating redox status. Biol Pharm Bull 34: 1072-1077, 2011.

12. Gurcan O, Gurcay AG, Kazanci A, Senturk S, Bodur E, Karaca EU, Turkoglu OF, Bavbek M: Effect of asiatic acid on the treatment of spinal cord injury: An Experimental study in rats. Turk Neurosurg 27: 259-264, 2017.

13. Mo YP, Yao HJ, Lv W, Song LY, Song HT, Yuan XC, Mao YQ, Jing QK, Shi SH and Li ZG: Effects of electroacupuncture at governor vessel acupoints on neurotrophin-3 in rats with experimental spinal cord injury. Neural Plast 2016: 2371875, 2016.

14. Armstrong HK, Koay YC, Irani S, Das R, Nassar ZD; Australian Prostate Cancer BioResource, Selth LA, Centenera MM, McAlpine SR and Butler LM: A novel class of Hsp90 C-terminal modulators have pre-clinical efficacy in prostate tumor cells without induction of a heat shock response. Prostate 76: 1546-1559, 2016.

15. Allen AR: Surgery of experimental lesion of spinal cord equivalent to crush injury of fracture dislocation of spinal column. J American Med Asso lvii: 878-880, 1962.

16. Allen AR and Reginald A: Remarks on the histopathological changes in the spinal cord due to impact. An experimental study. J Nervous Mental Dis 41: 141-147, 1914.

17. Basso DM, Beattie MS and Bresnahan JC: A sensitive and reliable locomotor rating scale for open field testing in rats. J Neurotrauma 12: 1-21, 1995. 
18. Livak KJ and Schmittgen TD: Analysis of relative gene expression data using real-time quantitative PCR and the 2(-Delta Delta C(T)) method. Methods 25: 402-408, 2001

19. Oh JH, Hyun JY and Varshavsky A: Control of Hsp90 chaperone and its clients by $\mathrm{N}$-terminal acetylation and the $\mathrm{N}$-end rule pathway. Proc Natl Acad Sci USA 114: E4370-E4379, 2017.

20. Krzemien-Ojak L, Goral A, Joachimiak E, Filipek A and Fabczak H: Interaction of a novel chaperone PhLP2A with the heat shock protein Hsp90. J Cell Biochem 118: 420-429, 2017.

21. Bryan HK, Olayanju A, Goldring CE and Park BK: The Nrf2 cell defence pathway: Keapl-dependent and -independent mechanisms of regulation. Biochem Pharmacol 85: 705-717, 2013.

22. Kang CH, Kim MJ, Seo MJ, Choi YH, Jo WS, Lee KT, Jeong YK and Kim GY: 5-Hydroxy-3,6,7,8,3'4'-hexamethoxyflavone inhibits nitric oxide production in lipopolysaccharide-stimulated BV2 microglia via NF- $\kappa \mathrm{B}$ suppression and Nrf-2-dependent heme oxygenase-1 induction. Food Chem Toxicol 57: 119-125, 2013.

23. Terazawa R, Akimoto N, Kato T, Itoh T, Fujita Y, Hamada N, Deguchi T, Iinuma M, Noda M, Nozawa Y and Ito M: A kavalactone derivative inhibits lipopolysaccharide-stimulated iNOS induction and $\mathrm{NO}$ production through activation of $\mathrm{Nrf} 2$ signaling in BV2 microglial cells. Pharmacol Res 71: 34-43, 2013.

24. Kim HJ, Zheng M, Kim SK, Cho JJ, Shin CH, Joe Y and Chung HT: CO/HO-1 induces NQO-1 expression via Nrf2 activation. Immune Netw 11: 376-382, 2011

25. Li WC, Jiang DM, Hu N, Qi XT, Qiao B and Luo XJ: Lipopolysaccharide preconditioning attenuates neuroapoptosis and improves functional recovery through activation of Nrf2 in traumatic spinal cord injury rats. Int J Neurosci 123: 240-247, 2013.

26. Li J, Johnson D, Calkins M, Wright L, Svendsen C and Johnson J: Stabilization of Nrf2 by tBHQ confers protection against oxidative stress-induced cell death in human neural stem cells. Toxicol Sci 83: 313-328, 2005

27. Rubiolo JA, Mithieux G and Vega FV: Resveratrol protects primary rat hepatocytes against oxidative stress damage: Activation of the Nrf2 transcription factor and augmented activities of antioxidant enzymes. Eur J Pharmacol 591: 66-72, 2008 .
28. Zhang J, Zhang Y, Liang C, Wang N, Zheng $\mathrm{H}$ and Wang J: Choline supplementation alleviates fluoride-induced testicular toxicity by restoring the NGF and MEK expression in mice. Toxicol Appl Pharmacol 310: 205-214, 2016.

29. Miller DM, Singh IN, Wang JA and Hall ED: Administration of the Nrf2-ARE activators sulforaphane and carnosic acid attenuates 4-hydroxy-2-nonenal-induced mitochondrial dysfunction ex vivo. Free Radic Biol Med 57: 1-9, 2013

30. Duan W, Zhang R, Guo Y, Jiang Y, Huang Y, Jiang H and Li C: Nrf2 activity is lost in the spinal cord and its astrocytes of aged mice. In Vitro Cell Dev Biol Anim 45: 388-397, 2009.

31. Ohashi R, Yan S, Mu H, Chai H, Yao Q, Lin PH and Chen C: Effects of homocysteine and ginsenoside Rb1 on endothelial proliferation and superoxide anion production. J Surg Res 133: 89-94, 2006.

32. Xie XS, Liu HC, Yang M,Zuo C, Deng Y and Fan JM: Ginsenoside $\mathrm{Rb} 1$, a panoxadiol saponin against oxidative damage and renal interstitial fibrosis in rats with unilateral ureteral obstruction. Chin J Integr Med 15: 133-140, 2009.

33. Sun Q, Meng QT, Jiang Y and Xia ZY: Ginsenoside Rb1 attenuates intestinal ischemia reperfusion induced renal injury by activating Nrf2/ARE pathway. Molecules 17: 7195-7205, 2012 .

34. Hwang YP and Jeong HG: Ginsenoside Rb1 protects against 6-hydroxydopamine-induced oxidative stress by increasing heme oxygenase-1 expression through an estrogen receptor-related PI3K/Akt/Nrf2-dependent pathway in human dopaminergic cells. Toxicol Appl Pharmacol 242: 18-28, 2010. 